\title{
A third order accurate Lagrangian finite element scheme for the computation of generalized molecular stress function fluids
}

\author{
Fasano, Andrea; Rasmussen, Henrik K.
}

Published in:

Journal of Non-Newtonian Fluid Mechanics

Link to article, DOI:

10.1016/j.jnnfm.2017.05.003

Publication date:

2017

Document Version

Peer reviewed version

Link back to DTU Orbit

Citation (APA):

Fasano, A., \& Rasmussen, H. K. (2017). A third order accurate Lagrangian finite element scheme for the computation of generalized molecular stress function fluids. Journal of Non-Newtonian Fluid Mechanics, 246, 1020. https://doi.org/10.1016/j.jnnfm.2017.05.003

\section{General rights}

Copyright and moral rights for the publications made accessible in the public portal are retained by the authors and/or other copyright owners and it is a condition of accessing publications that users recognise and abide by the legal requirements associated with these rights.

- Users may download and print one copy of any publication from the public portal for the purpose of private study or research.

- You may not further distribute the material or use it for any profit-making activity or commercial gain

- You may freely distribute the URL identifying the publication in the public portal 


\title{
A third order accurate Lagrangian finite element scheme for the computation of generalized molecular stress function fluids
}

\author{
Andrea Fasano \& Henrik Koblitz Rasmussen \\ Department of Mechanical Engineering, \\ Technical University of Denmark, DK-2800 Kgs. Lyngby, Denmark
}

\begin{abstract}
A third order accurate, in time and space, finite element scheme for the numerical simulation of three-dimensional time-dependent flow of molecular stress function type of fluids in a generalized formulation is presented. The scheme is an extension of the K-BKZ Lagrangian finite element method presented by Marín and Rasmussen (2009).
\end{abstract}

Keywords: Lagrangian, finite element, molecular stress function, polymer melt, break.

\section{Introduction}

The modelling of the flow behaviour of polymer melts is needed to evaluate the design of polymer processing operations. These involve shaping molten polymers into plastic products, enabling a production of key importance for our way of life.

The insight into the fluid dynamics of entangled liquids and polymer melts was initiated by phenomenologically based differential constitutive equations such as the Giesekus (1962) [1] and Phan-Thien and Tanner (1977) [2] models, whereas integral constitutive equations started with the K-BKZ $[3,4]$ model. A mathematical generalization of non-linear elasticity to viscolasticity. All these models are versatile constitutive equations that still are used to model flow of entangled polymer systems.

The understanding of the fluid dynamics of polymer melts still evolves $[5,6,7,8,9,10,11$, $12,13,14]$. This is a consequence of the developments in extensional rheometry $[15,16,17]$. In particular, the theoretical interpretation of measured extensional viscosities of idealised polymer systems $[18,19,20]$ has represented a challenging task. At least two of the previously referred constitutive equations seem to contain concepts capable of explaining the fluid mechanics of idealized entangled melt systems. Either considering monomeric friction [11, 12, 21, 22] or interchain pressure [14]. These theoretical efforts have been made aiming to predict the complex flow behaviour of entangled polymer systems in general, but particularly for polymer melts, driven by a need for accurate design of polymer production processes. Accurate design indeed requires knowledge of the flow behaviour of polymer melts. Moreover, polymer processing is geometrically complex, time dependent, and mostly complicated by the presence of moving melt surfaces or interfaces in three dimensions (3D). In the formulation of a numerical method for polymer melt flow, the choice of the particular constitutive equation to be implemented is important. Here we focus on the type of constitutive equation originally introduced by M.H. Wagner $[6,14]$. This molecular stress function constitutive equation is of the integral type. 
Computationally the Lagrangian finite element method $[23,24,25,26]$ is currently the only implemented technique for solving 3D time dependent flow problems with integral constitutive equations $[27,28,29,30]$. The formulation in [30] is capable of handling the molecular stress function constitutive equation, whereas all previous formulations are based on the K-BKZ model. Other steady [31] or unsteady [32, 33, 34, 35, 36, 37, 38] 2D formulations for flow of K-BKZ fluids have been published with a variety of numerical concepts. Especially for the molecular stress function type of constitutive equation, the only other published method is the 2D steady code by P. Olley and M.H. Wagner [39]. Note that, in a Lagrangian method, interface or surface movement is a natural boundary condition due to the particle formulation. Such a formulation is able to handle large displacements of surfaces or interfaces.

In this work the purpose is to develop a more accurate implementation of the three dimensional Lagrangian finite element method for flow of molecular stress function constitutive equation. The method in [30] converges second order with respect to spatial and time discretization. Both are increased to third order accuracy here.

\section{The stress tensor}

The stress tensor is described by a generalized molecular stress function model. This is an extension of the well established factorized K-BKZ model.

In the continuum mechanical analysis the deformation gradient field is defined - in Cartesian coordinates - by the components of the displacement gradient tensor as

$$
E_{i j}\left(\boldsymbol{x}, t, t^{\prime}\right)=\frac{\partial x_{i}}{\partial x_{j}^{\prime}}, \quad i=1,2,3 \text { and } j=1,2,3 .
$$

The coordinates $\boldsymbol{x}=\left(x_{1}, x_{2}, x_{3}\right)$ and $\boldsymbol{x}^{\prime}=\left(x_{1}^{\prime}, x_{2}^{\prime}, x_{3}^{\prime}\right)$ are the particle positions of the same particle at the present time $t$ and (all) the past time $t^{\prime}$, respectively. In a Lagrangian kinematics specification the independent variables are the initial particle positions of the particles $\boldsymbol{x}$ at the present time $t$. The dependent variables are consequently the positions of the particles $\boldsymbol{x}^{\prime}$ at the past times $t^{\prime}$. Bold symbols are used as matrix/vector notation in all formulas.

The Finger strain tensor is defined as follows

$$
\boldsymbol{B}\left(\boldsymbol{x}, t, t^{\prime}\right)=\boldsymbol{E}\left(\boldsymbol{x}, t, t^{\prime}\right) \cdot \boldsymbol{E}\left(\boldsymbol{x}, t, t^{\prime}\right)^{\dagger}
$$

where the symbol ' $\nmid$ ' denotes the transpose tensor operation.

Here we use the basic assumption of incompressibility allowing the third invariant of the Finger strain tensor to be unity: $I_{3}\left(\boldsymbol{x}, t, t^{\prime}\right)=\operatorname{det}\left[\boldsymbol{B}\left(\boldsymbol{x}, t, t^{\prime}\right)\right]=1$. We can further write that:

$$
\operatorname{det}\left[\boldsymbol{E}\left(\boldsymbol{x}, t, t_{0}\right)\right]=1
$$

which represents the equation of continuity. Furthermore, the first and second invariants are given as

$$
I_{1}\left(\boldsymbol{x}, t, t^{\prime}\right)=\operatorname{tr} \boldsymbol{B} \text { and } I_{2}\left(\boldsymbol{x}, t, t^{\prime}\right)=\operatorname{tr} \boldsymbol{B}^{-1}=\frac{1}{2}\left\{[\operatorname{tr} \boldsymbol{B}]^{2}-\operatorname{tr}[\boldsymbol{B} \cdot \boldsymbol{B}]\right\}
$$

The last equality is a consequence of the incompressibility and the Cayley-Hamilton theorem: $\boldsymbol{B}^{-1}=$ $\boldsymbol{B}^{2}-I_{1} \boldsymbol{B}+I_{2} \boldsymbol{\delta}$. The symbol ' -1 ' denotes the inverse tensor operation. Note that the dependence on $\left(\boldsymbol{x}, t, t^{\prime}\right)$ is shortened in the notation.

The isotropic strain tensors, $\boldsymbol{S}_{u}$, are defined as [40]

$$
\boldsymbol{S}_{u}\left(\boldsymbol{x}, t, t^{\prime}\right)=\psi_{1, u}\left(t-t^{\prime}, I_{1}, I_{2}\right)(\boldsymbol{\delta}-\boldsymbol{B})+\psi_{2, u}\left(t-t^{\prime}, I_{1}, I_{2}\right)\left(\boldsymbol{B}^{-1}-\boldsymbol{\delta}\right)
$$


were $u=1, \ldots, U . \quad \psi_{1, u}$ and $\psi_{2, u}$ are scalar functions that depend on the elapsed time and the invariants.

The stress tensor $\boldsymbol{\sigma}$ may be written as follows

$$
\boldsymbol{\sigma}=-\sum_{u=1}^{U} \int_{-\infty}^{t} M_{u}\left(t-t^{\prime}\right) f_{u}\left(\boldsymbol{x}, t, t^{\prime}\right)^{2} \boldsymbol{S}_{u}\left(\boldsymbol{x}, t, t^{\prime}\right) \mathrm{d} t^{\prime}
$$

in which $M_{u}\left(t-t^{\prime}\right)$ are the memory functions and $f_{u}$ denote scalar quantities referred to as the molecular stress functions. In the case of $f_{u}$ being unity, the above stress is identical to the well established factorized K-BKZ model [3, 4].

The molecular stress functions $f_{u}$, with an initial value $f_{u}\left(\boldsymbol{x}, t^{\prime}, t^{\prime}\right)=1$, are defined by using a set of differential equation in the present time $t$ as

$$
\frac{\partial}{\partial t} f_{u}\left(\boldsymbol{x}, t, t^{\prime}\right)=F_{u}\left(P_{1}, \ldots, P_{V}, \frac{\partial}{\partial t} P_{1}, \ldots, \frac{\partial}{\partial t} P_{V}, f_{1}\left(\boldsymbol{x}, t, t^{\prime}\right), \ldots, f_{U}\left(\boldsymbol{x}, t, t^{\prime}\right)\right)
$$

where $P_{v}\left(t-t^{\prime}, I_{1}\left(\boldsymbol{x}, t, t^{\prime}\right), I_{2}\left(\boldsymbol{x}, t, t^{\prime}\right)\right), v=1, \ldots, V$, are scalar functions depending on the first and second invariants, $I_{1}\left(\boldsymbol{x}, t, t^{\prime}\right)$ and $I_{2}\left(\boldsymbol{x}, t, t^{\prime}\right)$, and the relative time $t-t^{\prime}$.

\section{$3 \quad$ Finite element discretization}

Many of the details described in this section resemble the developments found in [30] and, most importantly, the present method is identical to the one from [29] in the case of $f_{u}\left(\boldsymbol{x}, t, t^{\prime}\right)=1$, i.e. the factorized K-BKZ model.

The equations of motion in the Lagrangian specification [41] may be formulated as follows

$$
\rho \frac{\partial^{2} \boldsymbol{x}}{\partial t^{2}}=\nabla \cdot(p \boldsymbol{\delta}-\boldsymbol{\sigma})+\rho \boldsymbol{g}
$$

here $\rho$ is the fluid density, $p$ is the pressure and $\boldsymbol{g}$ is the gravitational acceleration vector.

The discretization of the continuity equation (3) and of the equation of motion (8) follow the mixed Galerkin finite element method [42]. The Galerkin weak forms of the continuity equation and the momentum balance multiplied by arbitrary weight functions $\psi$ and $\phi$, respectively, follows the procedure by Rasmussen [27, 28]:

$$
\begin{gathered}
\int_{\Omega}\left[\operatorname{det}\left[\hat{\boldsymbol{E}}\left(\hat{\boldsymbol{x}}, t, t_{0}\right)\right]-1\right] \psi^{i} \mathrm{~d} \Omega=0 \\
\mathbf{0}=\int_{\Omega} \rho\left[\frac{\partial^{2} \hat{\boldsymbol{x}}}{\partial t^{2}}-\boldsymbol{g}\right] \phi^{j} \mathrm{~d} \Omega-\int_{\Omega} \hat{p} \boldsymbol{\nabla} \phi^{j} \mathrm{~d} \Omega+\int_{\Omega} \hat{\boldsymbol{\sigma}} \cdot \boldsymbol{\nabla} \phi^{j} \mathrm{~d} \Omega+\int_{\Gamma_{n}}[\boldsymbol{n} \cdot \hat{\boldsymbol{\pi}}] \phi^{j} \mathrm{~d} \Gamma_{n}
\end{gathered}
$$

where $i=1, \ldots, M$ and $j=1, \ldots, N . M$ represents the total number of pressure nodes and $N$ is the total number of coordinate nodes. $\Omega$ denotes the fluid domain, whereas $\Gamma_{n}$ are the surfaces with natural boundary conditions. $\boldsymbol{n}$ is the outward unit vector to the referred surface and the operator $\boldsymbol{\nabla}$ is the gradient operator. The approximated quantities of the exact variables are supplied with a hat. 
The particular case of the particle positions at the present time, $t$, is

$$
\hat{\boldsymbol{x}}=\sum_{i=1}^{N} \boldsymbol{x}^{i} \phi^{i}
$$

where the particle positions at any time, $t^{\prime}$, are approximated as

$$
\hat{\boldsymbol{x}}^{\prime}=\sum_{i=1}^{N} \boldsymbol{x}^{i}\left(\boldsymbol{x}^{i}\right) \phi^{i}
$$

The pressure field are approximated as

$$
\hat{p}(\hat{\boldsymbol{x}})=\sum_{n=1}^{M} p^{n}\left(\boldsymbol{x}^{n}\right) \cdot \psi^{n}
$$

The approximation of the particle positions is introduced directly into the displacement gradient tensor required to define the spatial discretization of the stress

$$
\hat{\boldsymbol{E}}\left(\hat{\boldsymbol{x}}, t, t^{\prime}\right)=\sum_{i=1}^{N} \boldsymbol{x}^{i} \nabla^{\prime} \phi^{i}
$$

where we have that:

$$
\nabla^{\prime}=\sum_{j=1}^{3} \delta_{j} \frac{\partial}{\partial x_{j}^{\prime}}
$$

Note that the shape functions are independent of time.

One issue is of particular importance. In a particle or Lagrangian specification the finite element mesh undergoes distortion during the flow. In the definition in (14) the coordinates at time $t$ and $t^{\prime}$ refer to the same finite element mesh. With the introduction of new meshes the general definition by Rasmussen [27, 28] replaces the gradient operator in (15) so that the displacement gradient tensor reads as

$$
\hat{\boldsymbol{E}}\left(\hat{\boldsymbol{x}}, t, t^{\prime}\right)=\sum_{i=1}^{N} \boldsymbol{x}^{i} \boldsymbol{\nabla}^{* \prime} \phi^{i}
$$

Equation (16) is indentical to equation (14) within one particular mesh.

As in Marín and Rasmussen [29], ten node quadratic tetrahedral interpolation functions $(\phi)$ are used for the spatial approximation, whereas four node linear interpolation functions $(\psi)$ are used within each tetrahedron for the pressure. The integration over the volume of a given element is carried out by a quadrature formula of degree 4 consisting of 11 points located inside the parent element [43]. This should ensure conservation of the expected third order spatial accuracy with the applied elements.

\subsection{Discretization of the time integral}

The stress from the constitutive equation is introduced directly into the Galerkin weak form of the momentum conservation equation (10). Then the deformation history in the integrals over $\Omega$ is evaluated numerically. (6) leads to

$$
-\int_{\Omega} \hat{\boldsymbol{\sigma}} \cdot \nabla \phi^{j} \mathrm{~d} \Omega=\int_{\Omega} \sum_{u=1}^{U} \int_{-\infty}^{t} M_{u}\left(t-t^{\prime}\right) \hat{f}_{u}\left(\boldsymbol{x}, t, t^{\prime}\right)^{2} \hat{\boldsymbol{S}}_{u}\left(\boldsymbol{x}, t, t^{\prime}\right) \mathrm{d} t^{\prime} \cdot \boldsymbol{\nabla} \phi^{j} \mathrm{~d} \Omega
$$


Keeping the present time $t$ fixed and splitting the time axis of the time integral in equation (17) leads to

$$
-\int_{\Omega} \hat{\boldsymbol{\sigma}} \cdot \nabla \phi^{j} \mathrm{~d} \Omega=\int_{\Omega} \sum_{u=1}^{U} \sum_{l=0}^{T} \int_{t_{l-1}}^{t_{l}} M_{u}\left(t-t^{\prime}\right) \hat{f}_{u}\left(\boldsymbol{x}, t, t^{\prime}\right)^{2} \hat{\boldsymbol{S}}_{u}\left(\boldsymbol{x}, t, t^{\prime}\right) \mathrm{d} t^{\prime} \cdot \boldsymbol{\nabla} \phi^{j} \mathrm{~d} \Omega
$$

Here the time axis is discretized at the following points

$$
t_{-1}=-\infty, \quad t_{0}=0, \quad t_{T}=t \quad \text { and } \quad t_{l-1}<t_{l}, \quad l=1,2, \ldots, T
$$

being the limits of the integral from $-\infty$ to 0 represented by $t_{-1}$ and $t_{0}$. In the initial condition at $t=t_{0}$, the location of all particles is known and assumed to be at rest until $t_{0}$. Therefore, the values of the approximated strains are constant in the time interval $t_{-1}$ to $t_{0}$. It is given that the strain at the present time, $t$, relative to the startup of flow at, $t_{0}, \hat{f}_{u}\left(\hat{\boldsymbol{x}}, t, t^{\prime}\right) \hat{\boldsymbol{S}}_{u}\left(\hat{\boldsymbol{x}}, t, t^{\prime}\right)=\hat{f}_{u}\left(\hat{\boldsymbol{x}}, t, t_{0}\right) \hat{\boldsymbol{S}}_{u}\left(\hat{\boldsymbol{x}}, t, t_{0}\right)$ for $t^{\prime} \leq t_{0}$.

The strain can be approximated by a quadratic interpolation function using $t^{\prime}=t_{l-2}, t_{l-1}$ and $t_{l}$ $($ for $l=2,3, \ldots, \mathrm{T})$

$$
\begin{aligned}
\hat{f}_{u}\left(\hat{\boldsymbol{x}}, t, t^{\prime}\right)^{2} \hat{\boldsymbol{S}}_{u}\left(\hat{\boldsymbol{x}}, t, t^{\prime}\right) & \approx \frac{t^{\prime}-t_{l-1}}{t_{l}-t_{l-1}} \hat{f}_{u}\left(\hat{\boldsymbol{x}}, t, t_{l}\right)^{2} \hat{\boldsymbol{S}}_{u}\left(\hat{\boldsymbol{x}}, t, t_{l}\right) \\
& -\frac{t^{\prime}-t_{l}}{t_{l}-t_{l-1}} \hat{f}_{u}\left(\hat{\boldsymbol{x}}, t, t_{l-1}\right)^{2} \hat{\boldsymbol{S}}_{u}\left(\hat{\boldsymbol{x}}, t, t_{l-1}\right) \\
& +\frac{\left(t^{\prime}-t_{l-1}\right)\left(t^{\prime}-t_{l}\right)}{\left(t_{l-1}-t_{l-2}\right)\left(t_{l}-t_{l-2}\right)} \hat{f}_{u}\left(\hat{\boldsymbol{x}}, t, t_{l-2}\right)^{2} \hat{\boldsymbol{S}}_{u}\left(\hat{\boldsymbol{x}}, t, t_{l-2}\right) \\
& +\frac{\left(t^{\prime}-t_{l-1}\right)\left(t^{\prime}-t_{l}\right)}{\left(t_{l}-t_{l-1}\right)\left(t_{l}-t_{l-2}\right)} \hat{f}_{u}\left(\hat{\boldsymbol{x}}, t, t_{l}\right)^{2} \hat{\boldsymbol{S}}_{u}\left(\hat{\boldsymbol{x}}, t, t_{l}\right) \\
& -\frac{\left(t^{\prime}-t_{l-1}\right)\left(t^{\prime}-t_{l}\right)}{\left(t_{l-1}-t_{l-2}\right)\left(t_{l}-t_{l-1}\right)} \hat{f}_{u}\left(\hat{\boldsymbol{x}}, t, t_{l-1}\right)^{2} \hat{\boldsymbol{S}}_{u}\left(\hat{\boldsymbol{x}}, t, t_{l-1}\right)
\end{aligned}
$$

These expansions are inserted in (17) so that (17) is approximated by

$$
\begin{aligned}
& -\int_{\Omega} \hat{\boldsymbol{\sigma}} \cdot \nabla \phi^{j} \mathrm{~d} \Omega \approx \\
& \sum_{u=1}^{U} \sum_{l=0}^{T-1}\left[M_{u, l, 1}-M_{u, l+1,0}+M_{u, l, 1,0}-M_{u, l+1,0,1}+M_{u, l+2,1,1}\right] \int_{\Omega} \hat{f_{u}}\left(\hat{\boldsymbol{x}}, t, t_{l}\right)^{2} \hat{\boldsymbol{S}}_{u}\left(\hat{\boldsymbol{x}}, t, t_{l}\right) \cdot \nabla \phi^{j} \mathrm{~d} \Omega
\end{aligned}
$$

Here the time integrals are defined as

$$
\begin{aligned}
M_{u, 0,1} & =\int_{t_{-1}}^{t_{0}} M_{u}\left(t-t^{\prime}\right) \mathrm{d} t^{\prime} \\
M_{u, l, p} & =\frac{1}{t_{l}-t_{l-1}} \int_{t_{l-1}}^{t_{l}} M_{u}\left(t-t^{\prime}\right)\left(t^{\prime}-t_{l-p}\right) \mathrm{d} t^{\prime} \text { and } \\
M_{u, l, p, q} & =\frac{1}{\left(t_{l-q}-t_{l-1-q}\right)\left(t_{l}-t_{l-1-p}\right)} \int_{t_{l-1}}^{t_{l}} M_{u}\left(t-t^{\prime}\right)\left(t^{\prime}-t_{l}\right)\left(t^{\prime}-t_{l-1}\right) \mathrm{d} t^{\prime}
\end{aligned}
$$


where $p=0,1, q=0,1, l=1,2, \ldots T$ and $u=1,2, \ldots, U$. The time discretization of the memory integrals is indentical to the ones defined in Marín and Rasmussen [29].

Notice, that we define $M_{u, l, p, q}=0$ for $l>T$ and $l \leq 1$. The latter restriction is equivalent to a linear interpolation in the first time step.

The time discretized weak form of the equation of motion (10) may be stated in the form

$$
\begin{aligned}
& -\sum_{u=1}^{U} \sum_{l=0}^{T-1}\left[M_{u, l, 1}-M_{u, l+1,0}+M_{u, l, 1,0}-M_{u, l+1,0,1}+M_{u, l+2,1,1}\right] \int_{\Omega} \hat{f}_{u}\left(\hat{\boldsymbol{x}}, t, t_{l}\right)^{2} \hat{\boldsymbol{S}}_{u}\left(\hat{\boldsymbol{x}}, t, t_{l}\right) \cdot \nabla \phi^{j} \mathrm{~d} \Omega \\
& +\int_{\Omega} \rho\left[\frac{\partial^{2} \hat{\boldsymbol{x}}}{\partial t^{2}}-\boldsymbol{g}\right] \phi^{j} \mathrm{~d} \Omega-\int_{\Omega} \hat{p} \boldsymbol{\nabla} \phi^{j} \mathrm{~d} \Omega+\int_{\Gamma_{n}}[\boldsymbol{n} \cdot \hat{\boldsymbol{\pi}}] \phi^{j} \mathrm{~d} \Gamma_{n}=\mathbf{0}
\end{aligned}
$$

for quadratic interpolation. The linear interpolation from Rasmussen [30] is recovered by setting $M_{u, l, p, q}=0$ for all $u, l, p$ and $q$ values. The applied quadratic interpolation increases the order of the time convergence from second to third, compared to the work by Rasmussen [27, 28, 30] and the third order approximation from [29] is regained for $f_{u}=1$.

\subsection{Time Discretization of the Molecular Stretch Function}

We need to determine the values of the molecular stress functions $f_{u}$ in the discretized points in time. The time axis is discretized at the following points

$$
t_{-1}=-\infty, \quad t_{0}=0, \quad t_{T}=t \quad \text { and } \quad t_{l-1}<t_{l}, \quad l=1,2, \ldots, T
$$

In the initial condition at $t=t_{0}$, the location of all particles is known and the fluid is assumed to be at rest until $t_{0}$. As a consequence, the values of the molecular stress functions are constants for past times $t^{\prime} \leq t_{0}$, namely for $t^{\prime} \in\left[t_{-1}: t_{0}\right]$.

In order to solve the differential equation defining the molecular stress functions $f_{u}$, we need to quantify the functions $\hat{P}_{v}\left(\hat{\boldsymbol{x}}, t, t^{\prime}\right) \equiv P_{v}\left(t-t^{\prime}, I_{1}\left(\boldsymbol{x}, t, t^{\prime}\right), I_{2}\left(\boldsymbol{x}, t, t^{\prime}\right)\right)$ in the present time step. The discretization in the present time of the scalar function $P_{v}$ in the current time step, relative to the 'creation' time $t_{l}$ by a quadratic interpolation function, is

$$
\begin{array}{r}
\hat{P}_{v}\left(\hat{\boldsymbol{x}}, t^{\prime \prime}, t_{l}\right) \approx \hat{P}_{v}\left(\hat{\boldsymbol{x}}, t, t_{l}\right)+\frac{t^{\prime \prime}-t}{t_{T}-t_{T-1}}\left[\hat{P}_{v}\left(\hat{\boldsymbol{x}}, t, t_{l}\right)-\hat{P}_{v}\left(\hat{\boldsymbol{x}}, t_{T-1}, t_{l}\right)\right] \\
+\frac{\left(t^{\prime \prime}-t_{T-1}\right)\left(t^{\prime \prime}-t_{T}\right)}{\left(t_{T-1}-t_{T-2}\right)\left(t_{T}-t_{T-2}\right)}\left[\hat{P}_{v}\left(\hat{\boldsymbol{x}}, t_{T-2}, t_{l}\right)-\hat{P}_{v}\left(\hat{\boldsymbol{x}}, t, t_{l}\right)\right. \\
\left.-\frac{t_{T-2}-t_{T}}{t_{T}-t_{T-1}}\left[\hat{P}_{v}\left(\hat{\boldsymbol{x}}, t, t_{l}\right)-\hat{P}_{v}\left(\hat{\boldsymbol{x}}, t_{T-1}, t_{l}\right)\right]\right]
\end{array}
$$

applied in the interval $t^{\prime \prime} \in\left[t_{T-1}: t\right]$, where $l=1,2, \ldots, T-1$. Note that a linear interpolation is required for $l=1$ due to the non-differentiability in time at $t_{0}$. The above quadratic interpolation of the scalar function $P$ is used in the current time interval between $t^{\prime \prime}=t_{T-1}$ and $t^{\prime \prime}=t_{T}=t$. It follows that the time derivative of the above scalar function in the present time (i.e. $t^{\prime \prime}$ ) is

$$
\begin{array}{r}
\frac{\partial}{\partial t^{\prime \prime}} \hat{P}_{v}\left(\hat{\boldsymbol{x}}, t^{\prime \prime}, t_{l}\right) \approx \frac{1}{t_{T}-t_{T-1}}\left[\hat{P}_{v}\left(\hat{\boldsymbol{x}}, t, t_{l}\right)-\hat{P}_{v}\left(\hat{\boldsymbol{x}}, t_{T-1}, t_{l}\right)\right] \\
+\frac{\left(t^{\prime \prime}-t_{T}\right)+\left(t^{\prime \prime}-t_{T-1}\right)}{\left(t_{T-1}-t_{T-2}\right)\left(t_{T}-t_{T-2}\right)}\left[\hat{P}_{v}\left(\hat{\boldsymbol{x}}, t_{T-2}, t_{l}\right)-\hat{P}_{v}\left(\hat{\boldsymbol{x}}, t, t_{l}\right)\right. \\
\left.-\frac{t_{T-2}-t_{T}}{t_{T}-t_{T-1}}\left[\hat{P}_{v}\left(\hat{\boldsymbol{x}}, t, t_{l}\right)-\hat{P}_{v}\left(\hat{\boldsymbol{x}}, t_{T-1}, t_{l}\right)\right]\right]
\end{array}
$$


The molecular stress function defined in equation (7) is solved by using an explicit integration method. Unlike the implicit method used in Rasmussen [30], this makes it possible to obtain a negligible error in the integration of equation (7). In the present study we apply a number, $K$, of classical explicit fourth order Runge-Kutta integration steps within one time step as follows

$$
\begin{aligned}
g_{u, i+1} & =g_{u, i}+\left(k_{u, 1}+2 k_{u, 2}+2 k_{u, 3}+k_{u, 4}\right) \cdot(\Delta t /(6 K)) \\
k_{u, 1} & =F_{u}\left(\boldsymbol{P}\left(\hat{\boldsymbol{x}}, t_{T-1}+i \Delta t / K, t_{l}\right), \frac{\partial}{\partial t} \boldsymbol{P}\left(\hat{\boldsymbol{x}}, t_{T-1}+i \Delta t / K, t_{l}\right), \boldsymbol{g}_{i}\right) \\
k_{u, 2} & =F_{u}\left(\boldsymbol{P}\left(\hat{\boldsymbol{x}}, t_{T-1}+(i+1 / 2) \Delta t / K, t_{l}\right), \frac{\partial}{\partial t} \boldsymbol{P}\left(\hat{\boldsymbol{x}}, t_{T-1}+(i+1 / 2) \Delta t / K, t_{l}\right), \boldsymbol{g}_{i}+\boldsymbol{k}_{1}(\Delta t /(2 K))\right) \\
k_{u, 3} & =F_{u}\left(\boldsymbol{P}\left(\hat{\boldsymbol{x}}, t_{T-1}+(i+1 / 2) \Delta t / K, t_{l}\right), \frac{\partial}{\partial t} \boldsymbol{P}\left(\hat{\boldsymbol{x}}, t_{T-1}+(i+1 / 2) \Delta t / K, t_{l}\right), \boldsymbol{g}_{i}+\boldsymbol{k}_{2}(\Delta t /(2 K))\right) \\
k_{u, 4} & =F_{u}\left(\boldsymbol{P}\left(\hat{\boldsymbol{x}}, t_{T-1}+(i+1) \Delta t / K, t_{l}\right), \frac{\partial}{\partial t} \boldsymbol{P}\left(\hat{\boldsymbol{x}}, t_{T-1}+(i+1) \Delta t / K, t_{l}\right), \boldsymbol{g}_{i}+\boldsymbol{k}_{3}(\Delta t / K)\right) \\
i & =0, \ldots, K-1
\end{aligned}
$$

in which $\Delta t=t-t_{T-1}=t_{T}-t_{T-1}, f_{u}\left(t_{T-1}, t_{l}\right)=g_{u, 0}$ and $f_{u}\left(t_{T}, t_{l}\right)=f_{u}\left(t, t_{l}\right)=g_{u, K}$. We have adopted the vector notation for $\boldsymbol{k}_{1}, \boldsymbol{k}_{2}, \boldsymbol{k}_{3}, \boldsymbol{g}_{i}$ and $\boldsymbol{P} . f_{u}\left(t_{T-1}, t_{l}\right)=g_{u, 0}$ are known from the previous time step and the initial value of $f_{u}\left(t_{l}, t_{l}\right)=1$, although in the case of the introduction of a new mesh all values have to be recalculated. Contrary to the work by Rasmussen [30], any needed accuracy can be achieved in this formulation. Further, due to this explicit formulation the molecular stress function, $f_{u}\left(t, t_{l}\right)$, is not a part of the equation system as in Rasmussen [30].

The non-linear system of equations contains 3N+M unknowns, which consist of $\mathrm{N}$ nodal points and $\mathrm{M}$ pressures found at each time step. We apply a Newton-Raphson iterative scheme to solve the non-linear system at each time step. In the Newton-Raphson scheme we use a parallel linear equation solver developed by Schenk and Gärtner [44, 45] (PARDISO) because of the large number of unknown variables involved.

\section{$4 \quad$ Numerical scheme testing}

In the development of a new numerical scheme it is essential to validate the implementation. In any new numerical scheme it is indeed necessary to make a thorough testing of the implementation to ensure both the correctness of the scheme and the code. The accuracy towards known solutions and previously published computations should be established. Also, the computational stability and especially the actual convergence order should be documented.

A variety of benchmark problems have been established in the area of viscoelastic fluid mechanics. For free surface flow of integral constitutive equations of the K-BKZ type, one of them has involved the original 2D axisymmetric steady extrusion swell of the IUPAC-LDPE melt [46, 47], whereas, for 3D time dependent flow, more recent studies by Bach et al. [48] have focused on modelling the stretching of a filament considering the growth of non-axisymmetric surface instabilities near the end plates. However, for more advanced viscoelastic models of the integral type a benchmark problem has not been established yet. Nevertheless, in this regard a few problems has been investigated $[39,49,50]$, two of which are relevant for the particular type of constitutive equation adopted here. They both consider the free surface evolution of the necking phenomena on a filament stretched [49] or under stretching [50], respectively.

Here we investigate the convergence in time and space of the proposed method on the necking phenomena during filament stretch [50] and compare the results with the simulations from [50], involving 2D axisymmetric flow modelling of the break of ideally monodisperse polymer melts during 
extension. The calculated break is a consequence of small initial sample imperfections. This problem is strictly axisymmetric with axial $(z)$ and radial $(r)$ coordinates. The boundary conditions on the free moving surface are no stress, pressure and surface tension. The initial shape is a cylinder with radius $R_{0}$ and length $L_{0}$. We impose symmetry at the center $(z=0)$ and at both ends of the samples $\left(z= \pm L_{0} / 2\right)$. An extensional flow is applied by an exponential extension of the axial coordinates at the ends of the cylinder (i.e. $z= \pm L / 2$ ). The length of the domain is defined as $L(t)=L_{0} \exp (\epsilon)$, where $L(t=0)=L_{0}$ is the initial length of the actual extended cylinder. With the assumption of a constant nominal strain rate $\dot{\epsilon}$, the nominal strain is $\epsilon=\dot{\epsilon} \cdot t$. The initial condition (at $t=0$ ) of the domain is stress free and at rest.

The deviation from the ideal cylindrical shape is a sinusoidally shaped suppression in the surface with a depth of $H$ and a width of $2 W$ in the axial direction. This small deviation on the free surface evolves into necking followed by a break during extension. A particular computation of the surface evolution showing the necking phase evolving into a break during extension is displayed in Figure 1. This is shown in a cylindrical coordinate system as there is ideally no variation in the azimuthal direction $(\theta)$. The finite element code here is formulated in 3D rectangular coordinates. To simulate the three-dimensional flow problem only one element (i.e. three nodes) is used in the azimuthal direction. The mesh is in three dimensions where symmetry is assumed at $\theta=0$ and $\theta=\theta_{0}$ in the simulations. The azimuthal angle is kept sufficiently small to avoid that is has a significant effect, so that each simulation can essentially be thought of as an ideal computation in a two-dimensional cylindrical coordinate system, $(r, z)$. The inset in Figure 1 shows the detail of the initial perturbation assumed on the sample surface. The computations are referred to using cylindrical coordinate system in the following sections, although they are basically are three-dimensional.

\subsection{Constitutive equation}

The constitutive equation used is identical to that described in [50]. It relies on Currie's [51] approximation of the Doi-Edwards reptation strain tensor based on the independent alignment assumption. The tensor is as follows

$$
\boldsymbol{S}_{\mathrm{DE}}=\frac{5}{I_{1}-1+2 \sqrt{I_{2}+13 / 4}}\left[(\boldsymbol{\delta}-\boldsymbol{B})+\frac{1}{\sqrt{I_{2}+13 / 4}}\left(\boldsymbol{B}^{-1}-\boldsymbol{\delta}\right)\right]
$$

Note that here we drop the dependence on $\left(\boldsymbol{x}, t, t^{\prime}\right)$ in the notation. As in [50], we apply the recent constitutive equation by Wagner et al. [6, 52], based on the 'interchain pressure' concept [5]. The components of the stress tensor $\left(\sigma_{\mathrm{ij}}\right)$ are the following:

$$
\begin{gathered}
\sigma_{i j}=\int_{-\infty}^{t} M\left(t-t^{\prime}\right) f\left(t, t^{\prime}\right)^{2} \boldsymbol{S}_{\mathrm{DE}} d t^{\prime} \\
\frac{\partial}{\partial t} f\left(t, t^{\prime}\right)=f\left(t, t^{\prime}\right)\left[\frac{\partial}{\partial t} P\left(I_{1}, I_{2}\right)-\frac{f\left(t, t^{\prime}\right)}{3 \tau_{R}}\left(f\left(t, t^{\prime}\right)^{3}-1\right)\right]
\end{gathered}
$$

in which

$$
P\left(I_{1}, I_{2}\right)=\frac{1}{2} \ln \left(\frac{I_{1}+2 \sqrt{I_{2}+13 / 4}-1}{7}\right) .
$$

The initial value of the molecular stress function is $f\left(t^{\prime}, t^{\prime}\right)=1$. $\tau_{R}$ is a time constant commonly referred to as the Rouse time. The memory function used is the BSW model (Baumgaertel, Schausberger and Winter [53]) without the glassy part:

$$
M\left(t-t^{\prime}\right)=\int_{0}^{\lambda_{\max }} n_{e} G_{N}^{0}\left(\frac{\lambda}{\lambda_{\max }}\right)^{n_{e}} \frac{e^{\left(t-t^{\prime}\right) / \lambda}}{\lambda^{2}} d \lambda .
$$




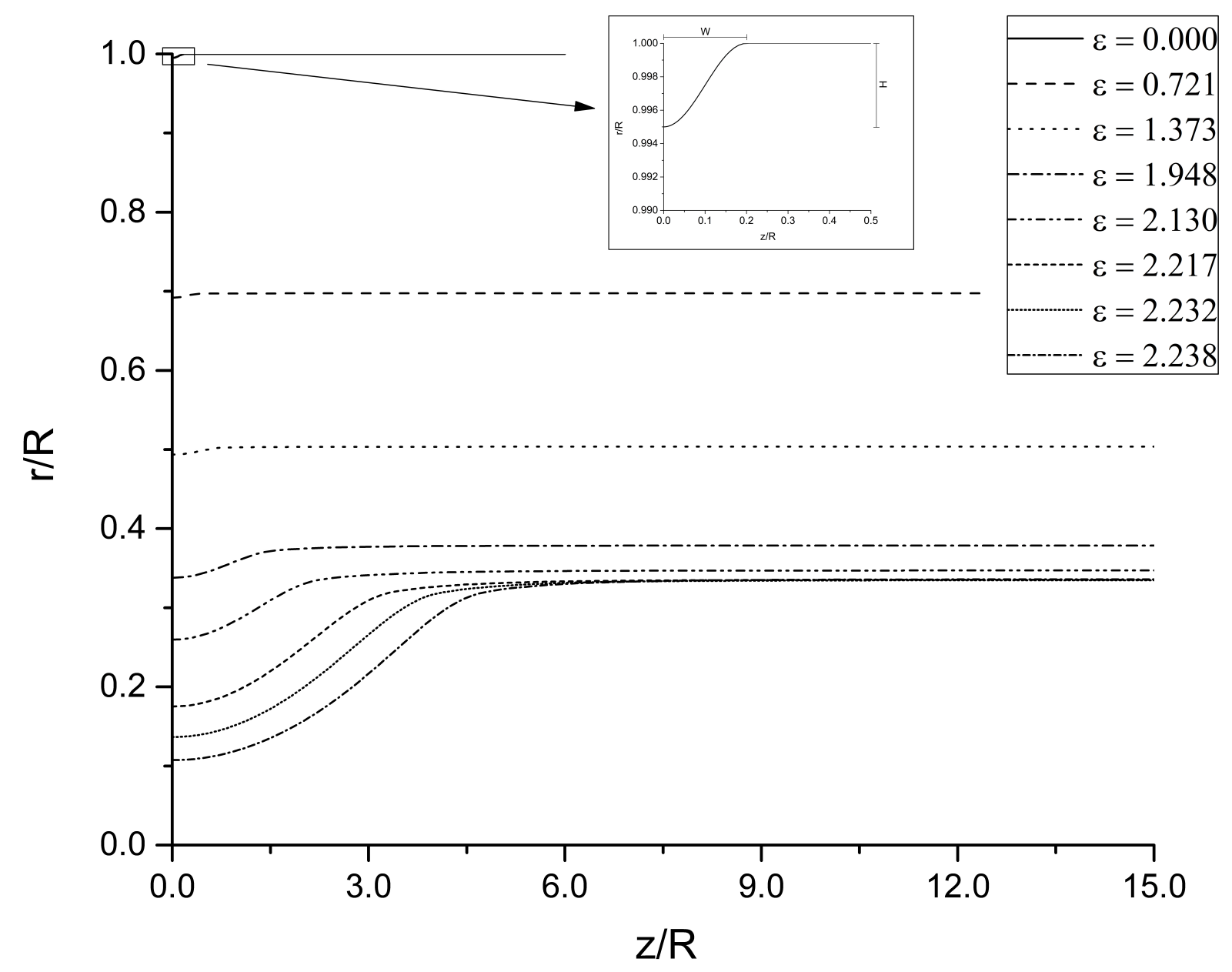

Figure 1: Dynamic development of the boundary contours $r$ as a function of the axial coordinate $z$ in a finite element simulation where $L_{0} / R=12$ and $\dot{\epsilon} \lambda_{\max }$ of 208.62. An initial perturbation of the cylindrical sample of $H / R=0.005$ and a width of $W / R=0.2$ are used. Both $\mathrm{x}$-axis and y-axis are referred to the initial radius $R$. The particular strain rate of $2.454353 \mathrm{~s}^{-1}$ represents a value around the strain softening-hardening transition, being close to the inverse of the Rouse time $\left(2.083333 \mathrm{~s}^{-1}\right)$. Inset: Sinusoidally shaped suppression in the surface with depth $H$ and width $2 W$ (along z-axis). Note that, as a consequence of the imposed symmetry conditions, an initial perturbation width of only $W$ and a sample length of only $L_{0} / 2$ are shown.

$\lambda_{\max }$ is the maximal relaxation time in the continuous distribution of the relaxation times and $G_{N}^{0}$ denotes the plateau modulus. The $n_{e}$ parameter has a unique value for each type of polymer. The parameters used here are: $\lambda_{\max }=85 \mathrm{~s}, G_{N}^{0}=870 \mathrm{kPa}, n_{e}=0.261$ and $\tau_{R}=0.48 \mathrm{~s}$.

\subsection{Breakup definition}

In Rasmussen [50] the flow dynamics was evaluated only as the critical strain, $\epsilon_{c}$, of break. A real break is of course defined as when the cross sectional area goes to zero. However, this limit cannot be reached exactly in a finite element implementation. To calculate a break within a continuum 
mechanical framework, Rasmussen [50] adopted the following definition: a break occurs if a linear extrapolation of the (smallest) cross sectional area of the sample, $A$, versus the time, $t$, reaches zero for a sufficiently small change in time. Numerically, $\epsilon_{c}$ was defined as that Hencky strain $\epsilon$ corresponding to the first time value at which the condition $A \cdot d \epsilon / d A<0.01 \cdot \epsilon$ was met. This criterion of course creates some degree of uncertainty on the evaluation of the break point. In particular, the breakup is expected to happen less than $1 \%$ later in terms of nominal strain.

\subsection{Time and spatial convergence}

The domain was discretized into a structured finite element mesh. We used meshes with the same connectivity throughout one particular simulation. This was expected to the best possible convergence behaviour.

In the spatial convergence test, different simulations varying the grid size were performed using the same time stepping. Remeshing was performed to ensure that the mesh did not undergo significant distorsion during a simulation. However, the number of elements, connectivities as well as surface node positions were the same before and after a remeshing operation. Moreover, remeshing and evaluation of the convergence order were performed at the same time values. Various grid sizes at the same strain rate $\dot{\epsilon}$ and initial perturbation depth $H / R$ were tested. When the onset of the necking phenomenon appeared, the time step size - and consequently also the remeshing intervals - was conveniently reduced in order to maintain the three-dimensional elements as undistorted as possible. In a Lagrangian formulation a node coordinate converges with reducing time step and mesh element size, both with an expected third order accuracy. We used the surface node positions to evaluate the convergence order, as they were the only ones being unaffected by a remeshing operation. The convergence tests were carried out using two (non-dimensional) strain rates: $\dot{\epsilon} \lambda_{\max }$ $=20.862$ and $\dot{\epsilon} \lambda_{\max }=834.48$ considering $H / R=0.015$, and $\dot{\epsilon} \lambda_{\max }=20.862$ and $\dot{\epsilon} \lambda_{\max }=579.50$ for $H / R=0.005$. With these two strain rate values the numerical tests considered rates both below and above the inverse Rouse time characterising the transition between strain softening and hardening, respectively. The least time resolved case as discussed below was applied. We performed the test on the first value of time where the criterion $A \cdot d \epsilon / d A<0.01 \cdot \epsilon$ was met, in the most spatially resolved computation. The roughest mesh consisted of 64 tetrahedra. Figure 2 shows how the mesh refinement was carried out. The grid size was decreased by a factor of 2 in each direction at each a mesh refinement. The refinement in the azimuthal direction was obtained by keeping the number of elements fixed in the azimuthal direction and decreasing the azimuthal angle by a factor of 2,4 and 8 , respectively. The number of elements shown in Figure 2 in the case of the more refined meshes was 288, 1216 and 4992, respectively. The meshes were structured and conveniently non-uniform, being finer near the sinusoidally shaped perturbation where the neck was expected to develop. To handle the remeshing, new structured meshes, with the same connectivity, were introduced to avoid progressing mesh distortion. For a structured mesh the computational accuracy between these meshes can be expected to decay as the inverse of the elements size used in a particular computation with the quadratic interpolation of the particle elements. Here the results are conveniently expressed in terms of normalized grid size, for which the error is proportional to grid size ${ }^{3}$. Figures $3 \mathrm{a}$ and $3 \mathrm{~b}$ show the error as function of the normalized grid size. Such error is defined as the average of the differences in surface node positions between the present simulation and a simulation with half of the grid size (i.e. refined twice as much in radial, axial and azimuthal directions). This error is normalized with respect to the initial radius $R$ of the cylindrical sample. The dashed lines $(-)$ represent the third order convergence. For the convergence in the spatial discretization the results indeed follow the expected third order slope corresponding to quadratic elements. Figures 4a, 4b and 4c display the initial, an intermediate and the final computational domain, respectively, in the highly spatial refined case (4992 tetrahedra) with a non-dimensional elongational rate $\dot{\epsilon} \lambda_{\max }$ of 20.862 for $H / R=0.005$. 


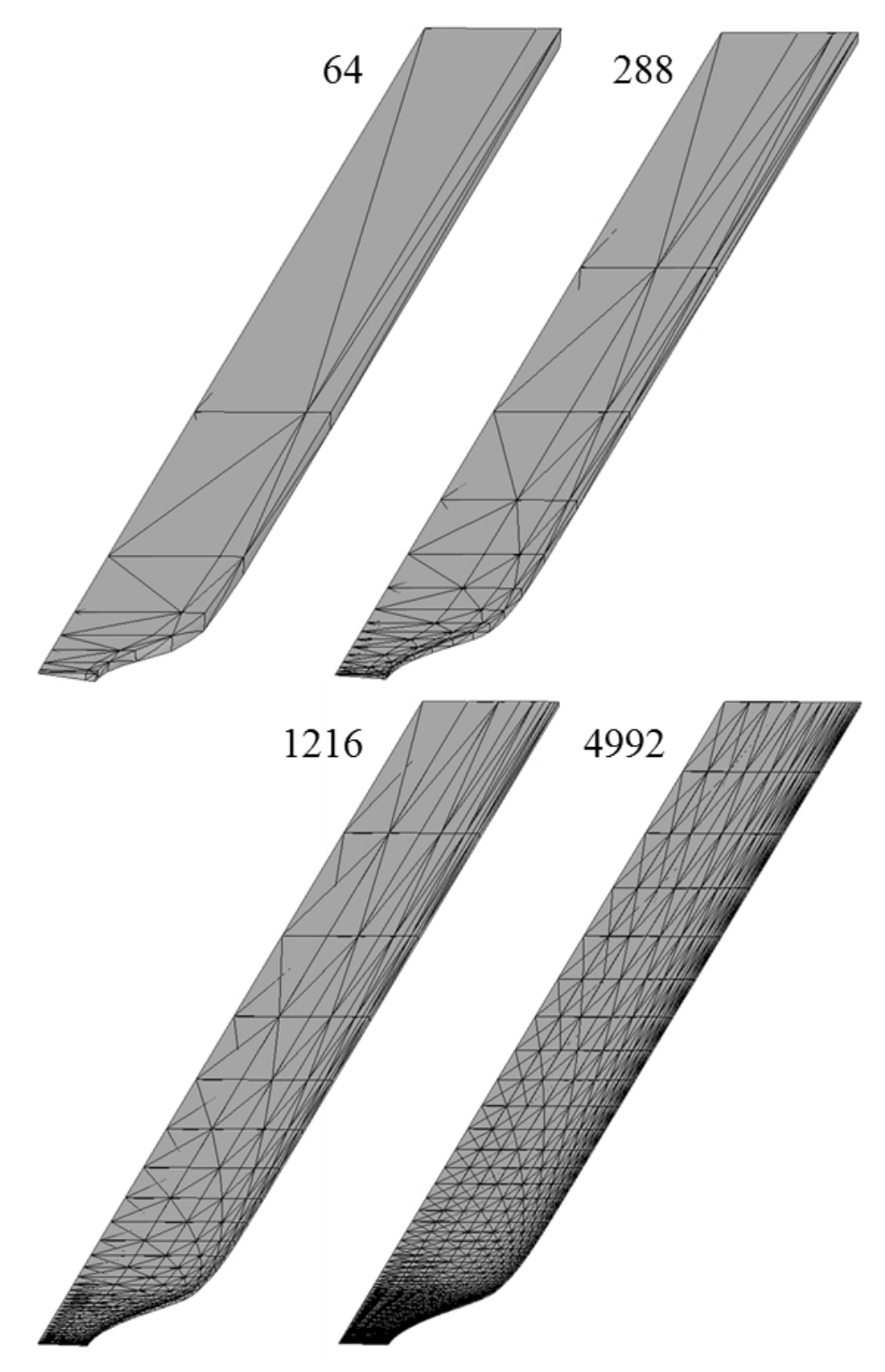

Figure 2: Three-dimensional mesh for different grid sizes for a non-dimensional elongational rate $\dot{\epsilon} \lambda_{\max }$ of 20.862. The initial perturbation has a depth of $H / R=0.015$ and a width of $W / R=0.2$, whereas the initial length of the sample is $L_{0} / R=12$. The roughest mesh consists of 64 tetrahedra. The mesh refinement in the azimuthal direction is obtained by keeping fixed the number of elements in the azimuthal direction and decreasing the azimuthal angle by a factor of 2,4 and 8 , respectively. The number of elements for the meshes are 64, 288, 1216 and 4992 tetrahedra, respectively. The snapshots are taken at the end of the respective simulations when the breakup criterion $A \cdot d \epsilon / d A<0.01 \cdot \epsilon$ is met for the most refined mesh. The software used for 3D mesh visualization is GMSH by Geuzaine and Remacle [54].

Similar to the spatial convergence tests, analyses were performed also for the time discretization. To test the time convergence, different computations varying the size of the time step were done using almost the same mesh. Here we split each time step into two equally sized (normalized time step of 0.500), and then four time steps in the most refined case (normalized time step size of 0.250), in order to reduce the time step size in a structured manner. We used a normalized grid size of 0.250 in all time convergence computations. The number of elements and nodes were the same as 

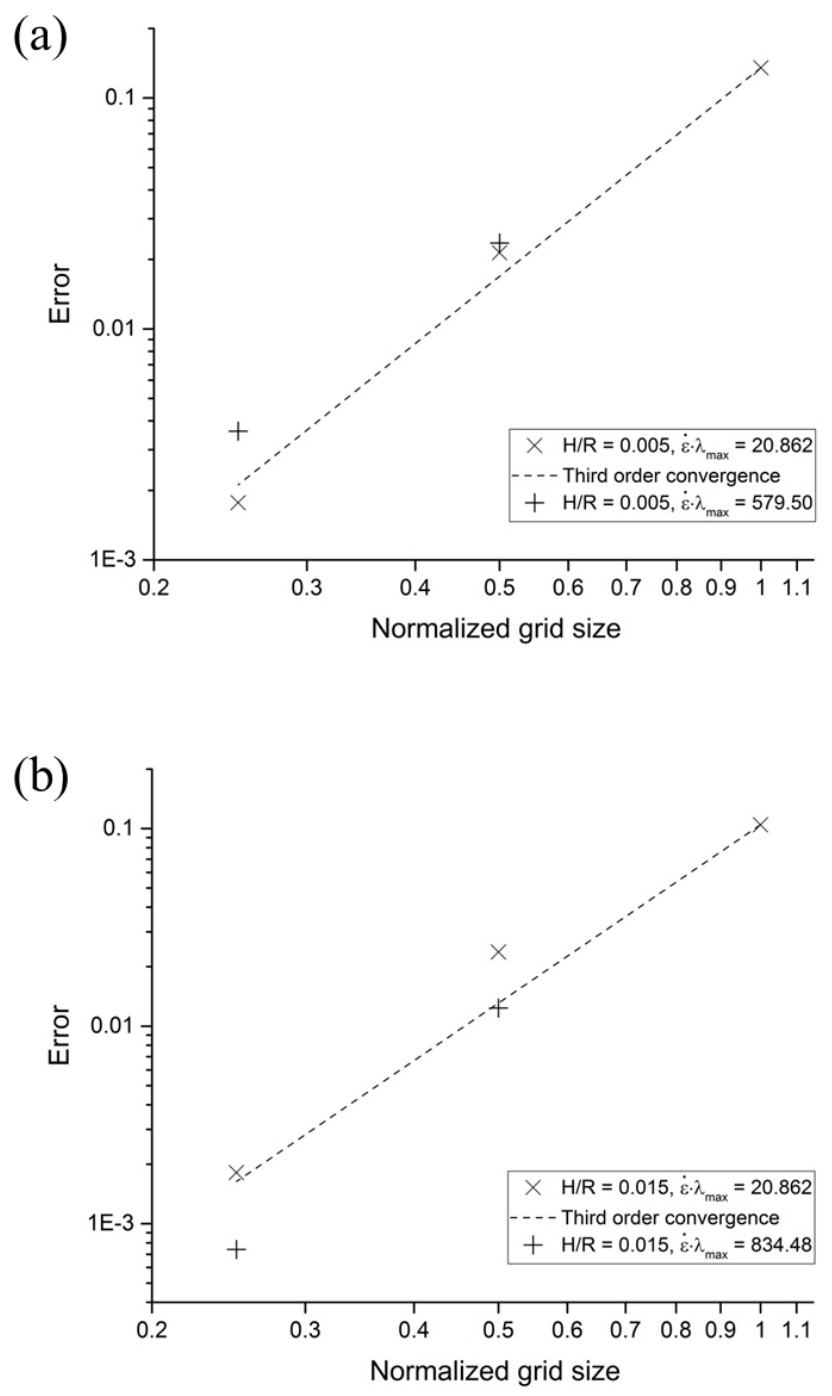

Figure 3: Convergence tests for various grid sizes. The y-axis shows the error in the computation of the surface node coordinates. Each point in the graph is calculated considering the average of the differences in surface node positions relative to a simulation using a mesh having a grid size two times smaller, in the spatial convergence test. Note that the error is non-dimensional as it is normalized with respect to the initial radius $R$ of the cylindrical sample. The initial perturbation has a width of $W / R=0.2$. The initial length of the sample is $L_{0} / R=12$. The dashed lines $(-)$ denote the third order convergence. a) Spatial convergence behaviour for an initial perturbation depth of $H / R=0.005$. Two strain rates, $\dot{\epsilon}=20.862 / 85 \mathrm{~s}^{-1}$ and $\dot{\epsilon}=579.50 / 85 \mathrm{~s}^{-1}$, are considered. b) Spatial convergence behaviour for an initial perturbation depth of $H / R=0.015$. Two different strain rate values, $\dot{\epsilon}=20.862 / 85 \mathrm{~s}^{-1}$ and $\dot{\epsilon}=834.48 / 85 \mathrm{~s}^{-1}$, are tested.

new simulations with different time stepping were carried out. In addition, remeshing procedures were performed at the same time values in a series of computations with varying time step size. In particular, remeshing was done every 4 time steps in the least time resolved computations. The simulations for the time convergence study were carried out at the same strain rates and strains as 
in the spatial convergence test. Figures $5 \mathrm{a}$ and $5 \mathrm{~b}$ show the error as a function of the normalized time step size. Similarly to the spatial convergence test, the error is defined as the average of the differences in surface node positions between the present simulation and a simulation having half of the time step size (that is, refined twice as much in time compared to the previous one), normalized with respect to $R$. Also in this case the results follow the third order accuracy as expected.

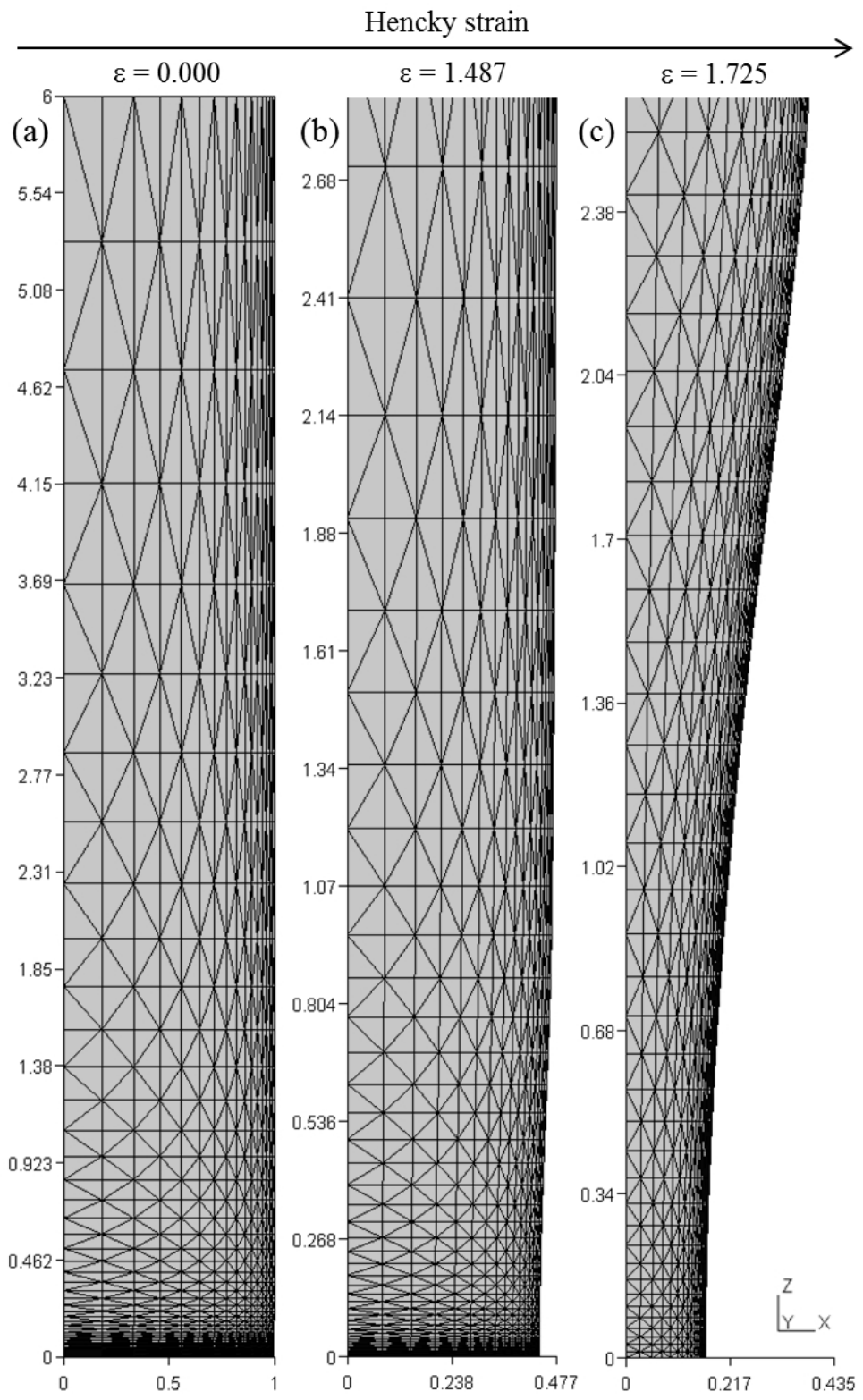

Figure 4: a) Initial, b) intermediate and c) final mesh used for the highly spatial refined simulation with a non-dimensional elongational rate $\dot{\epsilon} \lambda_{\max }$ of 20.862 . The number of tetrahedral elements is 4992. The initial length of the sample is $L_{0} / R=12$ and the initial perturbation has a depth of $H / R=0.005$ and a width of $W / R=0.2$.

\subsection{Breakup benchmarking}

The unambiguity in the definition of the critical strain in Rasmussen [50] was to be avoided here. Therefore, to obtain the critical strain we used the strict definition of $A \cdot d \epsilon / d A=0.01 \cdot \epsilon$. For an 

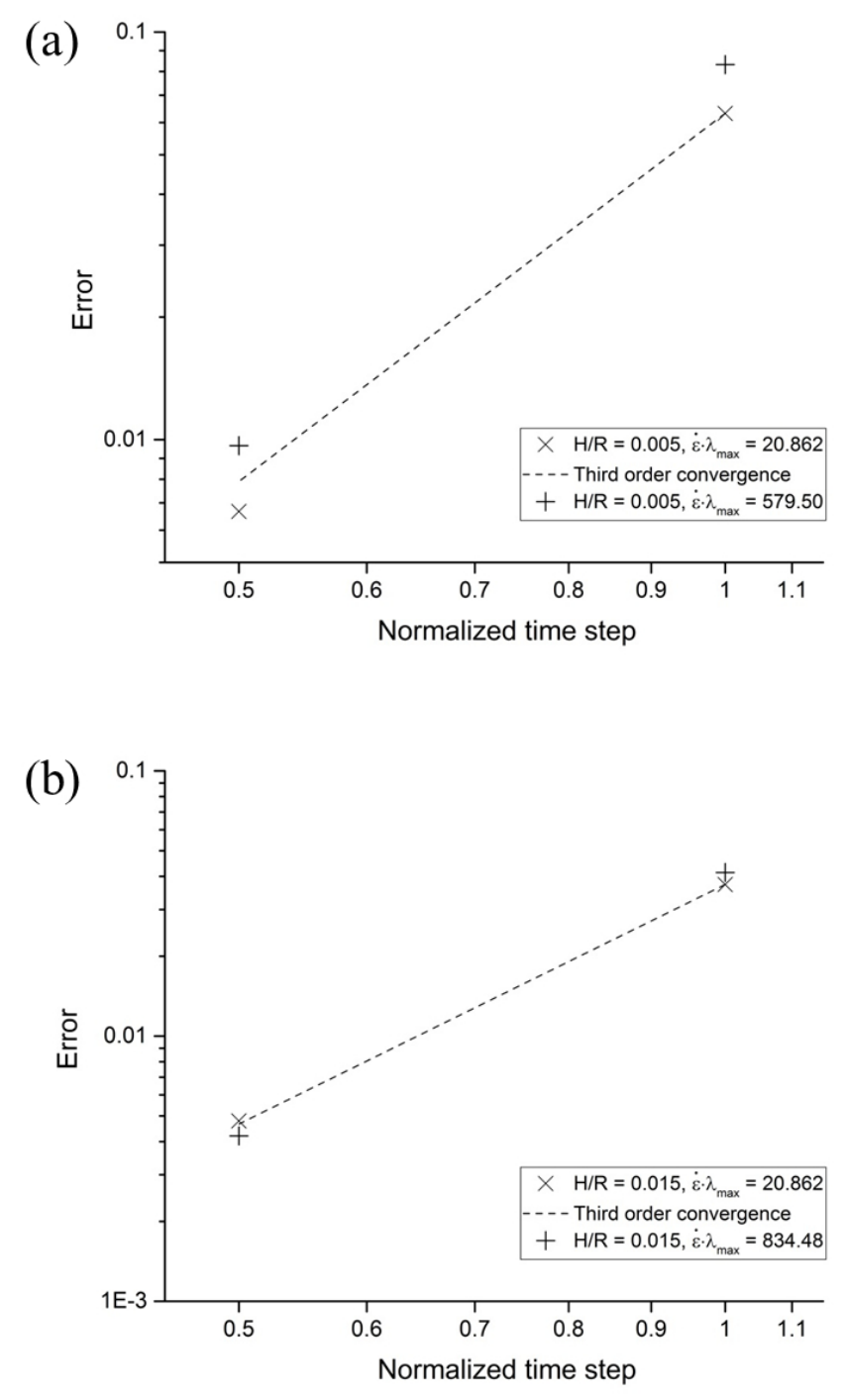

Figure 5: Convergence tests for different time steps. The y-axis shows the error in the computation of the surface node coordinates. Each point in the graph is calculated relative to a simulation having a time stepping being half of the previous one. The error is normalized with respect to the initial radius $R$ of the cylindrical sample. The initial length of the sample is $L_{0} / R=12$, whereas the initial perturbation width is $W / R=0.2$. The dashed lines $(-)$ indicate the third order convergence. a) Time convergence behaviour for an initial perturbation depth of $H / R=0.005$. Two strain rates, $\dot{\epsilon}$ $=20.862 / 85 \mathrm{~s}^{-1}$ and $\dot{\epsilon}=579.50 / 85 \mathrm{~s}^{-1}$, are considered. b) Time convergence behaviour for an initial perturbation depth of $H / R=0.005$. Two different strain rate values, $\dot{\epsilon}=20.862 / 85 \mathrm{~s}^{-1}$ and $\dot{\epsilon}=$ $834.48 / 85 \mathrm{~s}^{-1}$, are tested.

accurate evaluation of the critical strain $\left(\epsilon_{c}=\epsilon\right)$ we carried out a second order interpolation of the Hencky strain versus the calculated $(A / \epsilon) \cdot d \epsilon / d A$ values. The critical strain values could not be expected to follow any particular convergence orders, but convergence towards more accurate values with more resolved time and spatial resolution was expected.

Table 1 shows the computations carried out with fixed time steps changing the number of elements 
in the same way as discussed for the spatial convergence test. Both low $\left(\dot{\epsilon} \lambda_{\max }=20.862\right)$ and high $\left(\dot{\epsilon} \lambda_{\max }=834.48 / 85\right.$ for $H / R=0.015$ and $\dot{\epsilon} \lambda_{\max }=579.50$ for $\left.H / R=0.005\right)$ strain rate cases were considered. The increase in the accuracy in the evaluation of $\epsilon_{c}$ with decreasing grid size in the spatial discretization indicates that the computations with in the most spatial resolved case are accurate with five digits $( \pm 0.0001)$.

Table 1: Dependence of the critical strain $\epsilon_{c}$, defined by the criterion $A \cdot d \epsilon / d A=0.01 \cdot \epsilon$, on the normalized grid size. The asterisk indicates that the value reported for the roughest mesh in the case of $H / R=0.015$ and $\dot{\epsilon} \lambda_{\max }=834.48$ was extrapolated. This was necessary as the simulation could not meet the $A \cdot d \epsilon / d A=0.01 \cdot \epsilon$ criterion.

\begin{tabular}{crrrr}
\hline Grid size & $H / R=0.005$ & \multicolumn{2}{c}{$H / R=0.005$} & \multicolumn{2}{c}{$H / R=0.015$} & \multicolumn{2}{c}{$H / R=0.015$} \\
& $\dot{\epsilon} \lambda_{\max }=20.862$ & $\dot{\epsilon} \lambda_{\max }=579.50$ & $\dot{\epsilon} \lambda_{\max }=20.862$ & $\dot{\epsilon} \lambda_{\max }=834.48$ \\
& $\epsilon_{c}$ & $\epsilon_{c}$ & $\epsilon_{c}$ & $\epsilon_{c}$ \\
\hline \hline 1.000 & 1.7260 & 2.4431 & 1.4866 & $* 2.1994$ \\
\hline 0.500 & 1.7236 & 2.4481 & 1.4838 & 2.2042 \\
\hline 0.250 & 1.7233 & 2.4487 & 1.4833 & 2.2044 \\
\hline 0.125 & 1.7233 & 2.4488 & 1.4833 & 2.2044 \\
\hline
\end{tabular}

The analysis considering the effect of the reduction in the time stepping on $\epsilon_{c}$ was done exactly in the same way as discussed previously (Table 2). For these simulations a grid size of 0.250 was adopted. In Table 2) it may be seen that the least time resolved cases are accurate with four digits $( \pm 0.001)$.

Table 2: Dependence of the critical strain $\epsilon_{c}$, defined by the criterion $A \cdot d \epsilon / d A=0.01 \cdot \epsilon$, on the particular time stepping adopted in the computations. Similar to the spatial convergence test, this analysis considers the reduction of the time step size in a structured way, i.e. $1.000,0.500$ and 0.250 in terms of normalized time step.

\begin{tabular}{crrrr}
\hline Time step & $H / R=0.005$ & $H / R=0.005$ & $H / R=0.015$ & \multicolumn{2}{c}{$H / R=0.015$} \\
& $\dot{\epsilon} \lambda_{\max }=20.862$ & $\dot{\epsilon} \lambda_{\max }=579.50$ & $\dot{\epsilon} \lambda_{\max }=20.862$ & $\dot{\epsilon} \lambda_{\max }=834.48$ \\
& $\epsilon_{c}$ & $\epsilon_{c}$ & $\epsilon_{c}$ & $\epsilon_{c}$ \\
\hline \hline 1.000 & 1.7233 & 2.4487 & 1.4833 & 2.2044 \\
\hline 0.500 & 1.7239 & 2.4502 & 1.4836 & 2.2047 \\
\hline 0.250 & 1.7236 & 2.4499 & 1.4835 & 2.2045 \\
\hline
\end{tabular}

We further performed a series of simulations considering a broader range of strain rates. Again, an initial perturbation with width $W / R=0.2$ and depth $H / R=0.005$ and $H / R=0.015$, respectively, were applied, whereas the initial length of the sample was $L_{0} / R=12$. The computations of the critical strain were all done with the least time resolution but the most refined spatial resolution. Also here we adopted the stricter criterion $A \cdot d \epsilon / d A=0.01 \cdot \epsilon$ compared to the original one by Rasmussen [50]. The results from the computations are summarized in Table 3. Overall we expect these computations to have four reliable digits, namely $\epsilon_{c} \pm 0.001$.

Figure 6 shows the comparison between our strains at break and the calculations by Rasmussen [50]. The deviations from Rasmussen were below $2.6 \%$. Rasmussen [50] reported the strains at break with a discrepancy of one percent in the prediction of the actual break value. The overall accuracy of these simulations was within $3.5 \%$. This is expected to be a sufficient accuracy for comparison with experiments, which was the purpose in the work by Rasmussen [50]. 
Table 3: Break values $\epsilon_{c}( \pm 0.001)$ for an initial perturbation having a depth of $H / R=0.005$ and $H / R=0.015$, respectively. The initial length of the sample is $L_{0} / R=12$ and the initial perturbation has a width of $W / R=0.2$. The critical strain $\epsilon_{c}$ is defined by the criterion $A \cdot d \epsilon / d A=0.01 \cdot \epsilon$.

\begin{tabular}{rrrr}
\hline \multicolumn{2}{c}{$H / R=0.005$} & \multicolumn{2}{c}{$H / R=0.015$} \\
$\dot{\epsilon} \lambda_{\max }$ & $\epsilon_{c}$ & $\dot{\epsilon} \lambda_{\max }$ & $\epsilon_{c}$ \\
\hline \hline 1.44875 & 2.165 & 1.44875 & 1.742 \\
\hline 4.17240 & 1.668 & 4.17240 & 1.421 \\
\hline 20.8620 & 1.723 & 20.8620 & 1.483 \\
\hline 65.0000 & 1.961 & 65.0000 & 1.675 \\
\hline 208.620 & 2.229 & 208.620 & 1.914 \\
\hline 579.500 & 2.449 & 834.480 & 2.204 \\
\hline
\end{tabular}

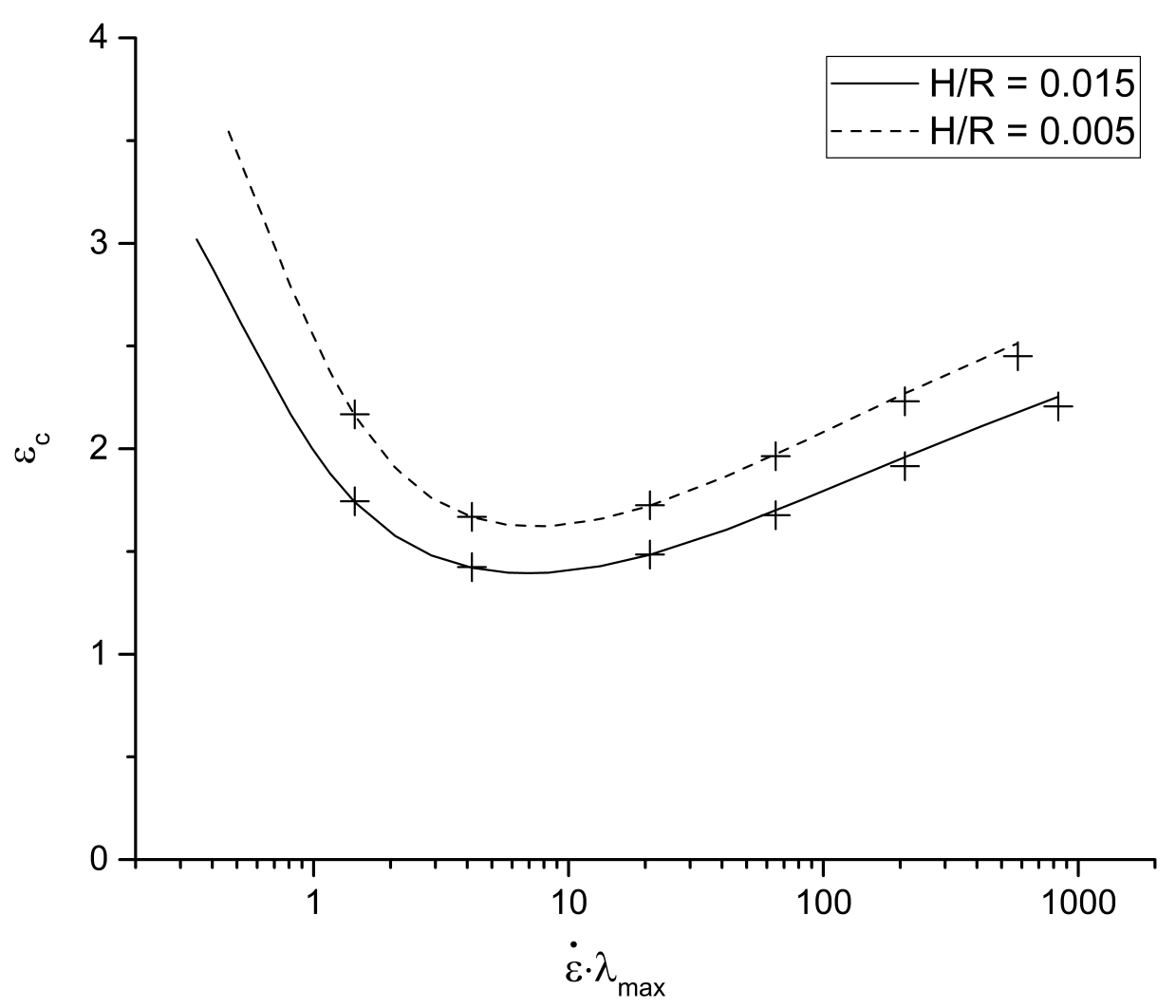

Figure 6: Strain of break $\epsilon_{c}$ as a function of the non-dimensional elongational rate $\dot{\epsilon} \lambda_{\max }$. Comparison of the present numerical method $(+)$ with the previous simulations reported in Rasmussen [50]. The break points are calculated for a cylindrical sample of length $L_{0} / R=12$, extended with fixtures moving in the axial direction. Initial perturbations of the cylindrical sample of depth $H / R=0.015$ and $H / R=0.005$ (solid and dashed lines, respectively) and a fixed width of $W / R=0.2$ are used.

From Figure 1 it may be seen that the necking develops very fast in time/nominal strain once it starts. The case considered here was $H / R=0.005$ and strain rate of $2.454353 \mathrm{~s}^{-1}$. This particular 
strain rate value was chosen as it is near the strain softening-hardening transition, corresponding approximately to the inverse of the Rouse time $\left(2.083333 \mathrm{~s}^{-1}\right)$. A high time and space resolution as well were used in the computation. The simulation was performed without using the $A \cdot d \epsilon / d A=0.01 \cdot \epsilon$ stopping criterion in order to follow the development of the surface also at strains very close to the actual break point. Remeshing operations were done more frequently in order to cope with the fast neck growth rate in the very high Hencky strain region. The final surface before breakup is shown at a Hencky strain of 2.238 , i.e. beyond the value of 2.229 from Table 3 where the stopping criterion was used. $\epsilon=2.238$ corresponds to a relative neck radius $r_{n e c k} / R$ of approximately 0.1 , after which the surface develops into a break extremely rapidly. The same can also be seen in Figure 7 , where the radius profile versus nominal strain is presented and compared with the ideal case (no initial perturbation). The two curves are overlapped until around a Hencky strain of 1 . After $\epsilon \simeq 1$ the $H / R=0.005$ curve deviates from the ideal one significantly, and for $\epsilon>2$ it decreases strongly non-linear as a consequence of the break point becoming closer and closer. Given the very high neck growth rate in the vicinity of the break, it was more difficult to obtain similar information from the engineering stress. As an example, Figure 8 displays the engineering stress as a function of the nominal stress in the non-ideal $(H / R=0.005)$ and ideal cases. The two curves indeed show a more pronounced overlap until near the breakup point.

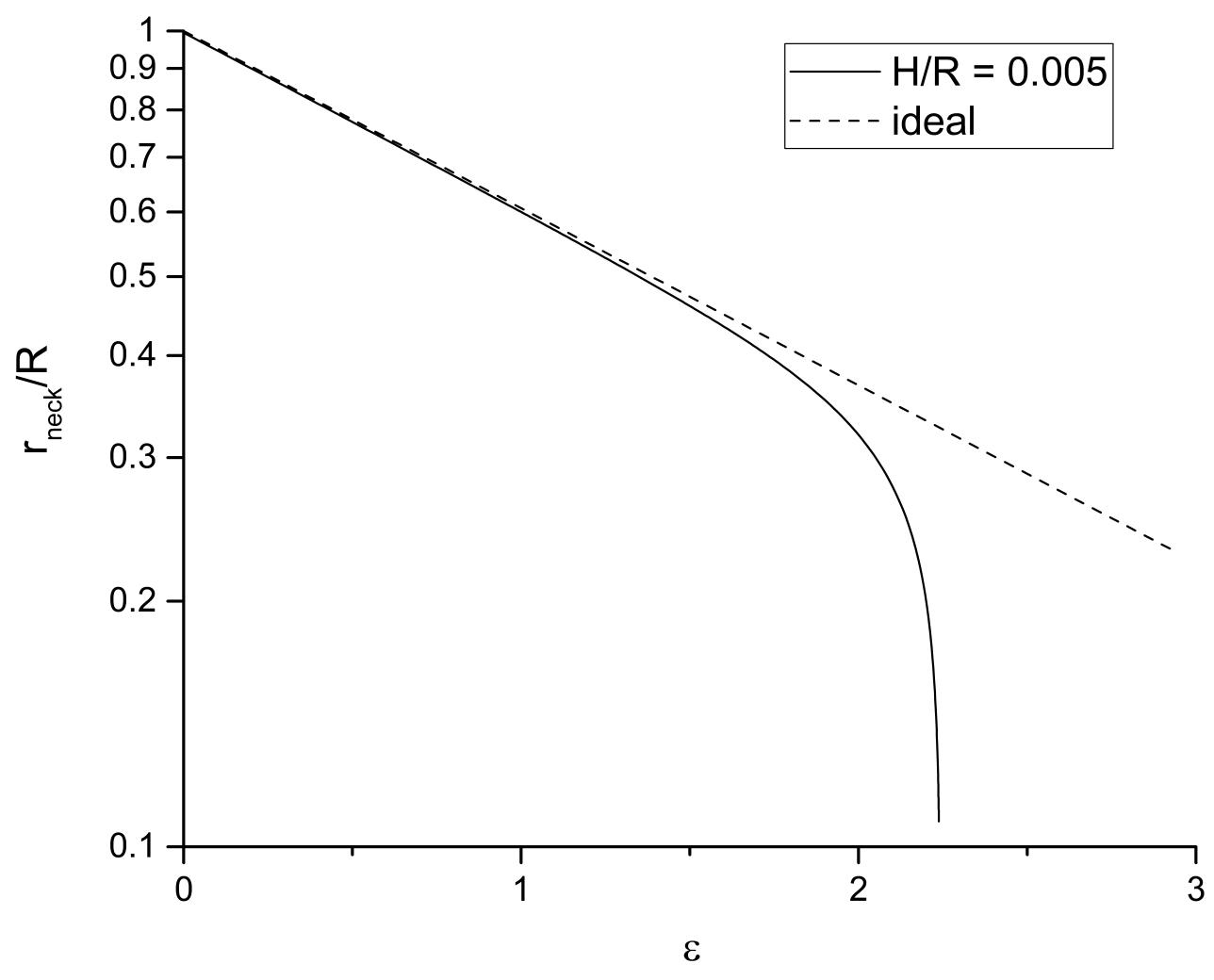

Figure 7: Neck radius $r_{n e c k}$, defined as the surface position at $z=0$, as a function of Hencky strain for the case $H / R=0.005$ and $W / R=0.2$ compared to the ideal case (no initial perturbation). The non-dimensional elongational rate $\dot{\epsilon} \lambda_{\max }$ is 20.862 . The $\mathrm{x}$-axis values are referred to relative to the initial radius $R$. The initial length of the cylindrical sample is $L_{0} / R=12$ 


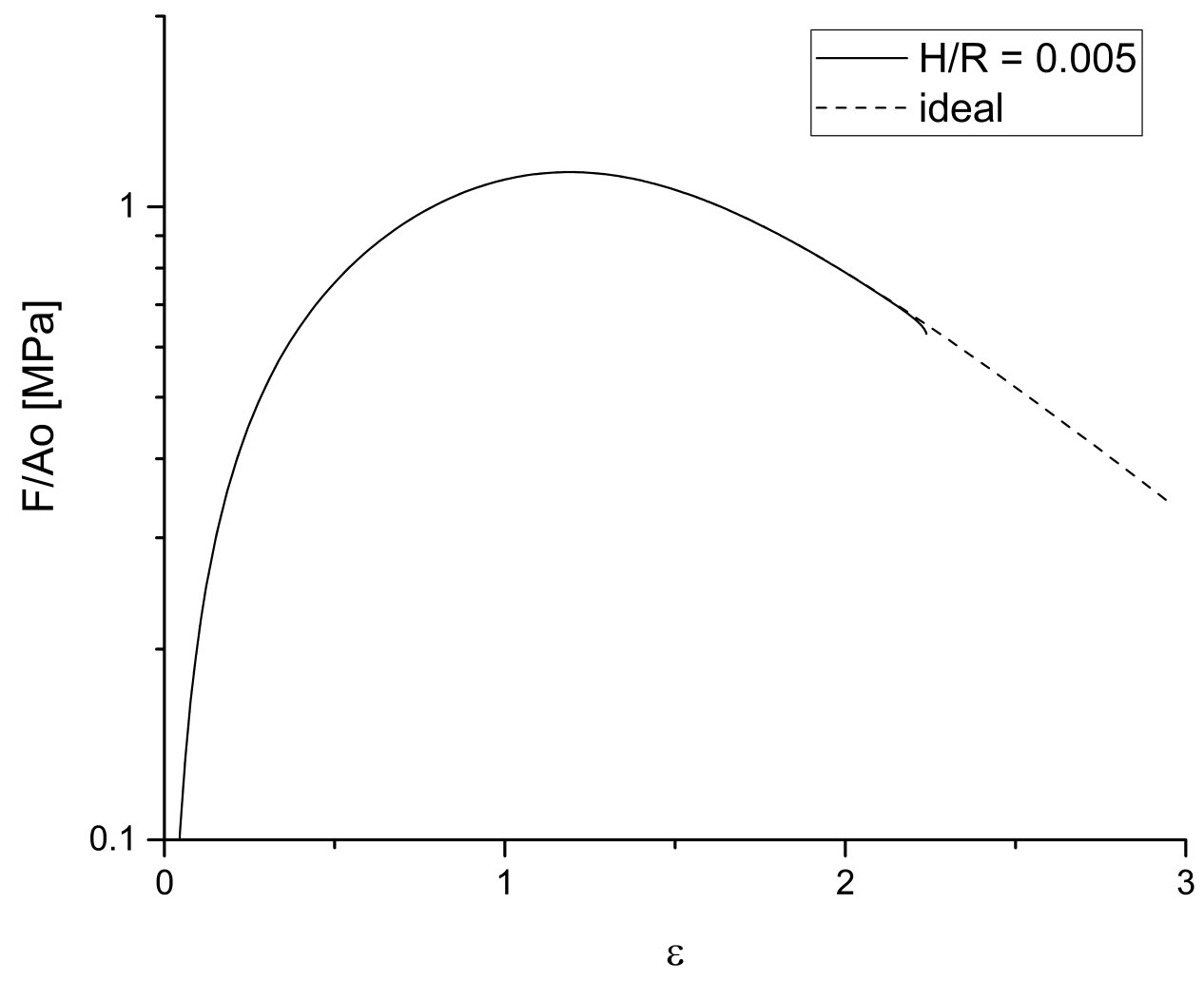

Figure 8: Dependence of the engineering stress, calculated as the force $F$ exerted by the fluid divided by the end-plate initial area $A_{0}$, on the Hencky strain $\epsilon$. The initial length of the sample $L_{0} / R$ and the non-dimensional elongational rate $\dot{\epsilon} \lambda_{\max }$ are 12 and 208.62, respectively. The initial perturbation of the cylindrical sample is $H / R=0.005$ and the perturbation width is $W / R=0.2$.

\section{Conclusions}

In this work a Galerkin finite element method for the simulation of three-dimensional transient flow of generalized molecular stress function type of fluids was developed. A Lagrangian kinematics description in Cartesian coordinates was used in a continuum mechanical framework. Ten node quadratic tetrahedral elements and linear four node tetrahedral elements were used for spatial discretization and pressure approximation, respectively. This ensured third order accuracy in the spatial discretization. The quadratic interpolation in the discretization of the time integral for a K-BKZ type of equation from Marin and Rasmussen [29] was extended to a generalized molecular stress function type of fluids. The third order convergence both in time and space of the finite element scheme was confirmed on the problem of the break of a filament undergoing extension. Agreement of the simulations using our code and the ones by Rasmussen [50] was demonstrated.

\section{Acknowledgements}

This work was supported by the People Programme (Marie Curie Actions) of the European Union's Seventh Framework Programme FP7/2007-2013/ under REA grant agreement number 608382. 


\section{References}

[1] H. Giesekus, Elasto-viskose Flüssigkeiten, für die in stationären Schichtrömungen sämtliche Normalspannungskomponenten verschieden gross sind, Rheologica Acta 2, 50-62 (1962).

[2] N. Phan-Thien, R.I. Tanner, A new constitutive equation derived from network theory, Journal of Non-Newtonian Fluid Mechanics 2, 353-365 (1977).

[3] A. Kaye. College of Aeronautics, Cranheld, Note no. 134, 1962.

[4] B. Bernstein, E.A. Kearsley, and L.J. Zapas, Study of stress relaxation with finite strain, Transactions of the Society of Rheology 7, 391-410 (1963).

[5] G. Marrucci and G. Ianniruberto, Interchain pressure effect in extensional flows of entangled polymer melts, Macromolecules 37, 3934-3942 (2004).

[6] M.H. Wagner, S. Kheirandish, and O. Hassager, Quantitative prediction of transient and steadystate elongational viscosity of nearly monodisperse polystyrene melts, Journal of Rheology 49, $1317-1327$ (2005).

[7] J.D. Schieber, D.M. Nair, and T. Kitkrailard, Comprehensive comparisons with nonlinear flow data of a consistently unconstrained Brownian slip-link model, Journal of Rheology 51, 11111141 (2007).

[8] S. Dhole, A. Leygue, C. Bailly, and R. Keunings, A single segment differential tube model with interchain tube pressure effect, Journal of Non-Newtonian Fluid Mechanics 161, 10-18 (2009).

[9] D. Auhl, P. Chambon, T.C.B. McLeish, and D.J. Read, Elongational flow of blends of long and short polymers: effective stretch relaxation time, Physical Review Letters 103, 136001 (2009).

[10] D.J. Read, K. Jagannathan, S.K. Sukumaran, and D. Auhl, A full-chain constitutive model for bidisperse blends of linear polymers, Journal of Rheology 56, 823-873 (2012).

[11] T. Yaoita, T. Isaki, Y. Masubuchi, H. Watanabe, G. Ianniruberto, and G. Marrucci, Primitive Chain Network Simulation of Elongational Flows of Entangled Linear Chains: Stretch/Orientation-induced Reduction of Monomeric Friction, Macromolecules 45, 2773-2782 (2012).

[12] G. Ianniruberto, A. Brasiello, and G. Marrucci, Simulations of fast shear flows of PS oligomers confirm monomeric friction reduction in fast elongational flows of monodisperse PS melts as indicated by rheooptical data, Macromolecules 45, 8058-8066 (2012).

[13] H.K. Rasmussen and Q. Huang, Rheologica Acta 53, 199-208 (2014).

[14] M.H. Wagner, Scaling relations for elongational flow of polystyrene melts and concentrated solutions of polystyrene in oligomeric styrene, Rheologica Acta 53, 765-777 (2014).

[15] T. Sridhar, V. Tirtaatmadja, D.A. Nguyen, and R.K. Gupta, Measurements of extensional viscosity of polymer solutions, Journal of Non-Newtonian Fluid Mechanics 40, 271-280 (1991).

[16] A. Bach, H.K. Rasmussen, and O. Hassager, Extensional viscosity for polymer melts measured in the filament stretching rheometer, Journal of Rheology 47, 429-441 (2003).

[17] M.L. Sentmanat, Miniature universal testing platform: from extensional melt rheology to solidstate deformation behavior, Rheologica Acta 43, 657-669 (2004). 
[18] P.K. Bhattacharjee, J.P. Oberhauser, G.H. McKinley, L.G. Leal, and T. Sridhar, Extensional rheometry of entangled solutions, Macromolecules 35, 10131-10148 (2002).

[19] A. Bach, K. Almdal, H.K. Rasmussen, and O. Hassager, Elongational viscosity of narrow molar mass distribution polystyrene, Macromolecules 36, 5174-5179 (2003).

[20] Q. Huang, O. Mednova, H.K. Rasmussen, N.J. Alvarez, A.L. Skov, K. Almdal, and O. Hassager, Concentrated Polymer Solutions are Different from Melts: Role of Entanglement Molecular Weight, Macromolecules 46, 5026-5035 (2013).

[21] Y. Masubuchi, Y. Matsumiya, and H. Watanabe, Test of Orientation/Stretch-Induced Reduction of Friction via Primitive Chain Network Simulations for Polystyrene, Polyisoprene, and Poly(n.butyl acrylate), Macromolecules 47, 6768-6775 (2014).

[22] G. Ianniruberto, Extensional flows of solutions of entangled polymers confirm reduction of friction coefficient, Macromolecules 48, 6306-6312 (2015).

[23] O. Hassager and C. Bisgaard, A finite-element code for initial-value probles with a Maxwell model, Rheologica Acta 21, 534-536 (1982).

[24] O. Hassager and C. Bisgaard. A Lagrangian finite-element method for the simulation of flow of non-newtonian liquids. Journal of Non-Newtonian Fluid Mechanics 12, 153-164 (1983).

[25] H.K. Rasmussen and O. Hassager, Simulation of transient viscoelastic flow, Journal of NonNewtonian Fluid Mechanics 46, 289-305 (1993).

[26] H.K. Rasmussen and O. Hassager. Simulation of transient viscoelastic flow with 2nd-order time integration. Journal of Non-Newtonian Fluid Mechanics 56, 65-84 (1995).

[27] H.K. Rasmussen. Time-dependent finite-element method for the simulation of three-dimensional viscoelastic flow with integral models, Journal of Non-Newtonian Fluid Mechanics 84, 217-232 (1999).

[28] H.K. Rasmussen, Lagrangian viscoelastic flow computations using Rivlin-Sawyers constitutive model, Journal of Non-Newtonian Fluid Mechanics 92, 227-243 (2000).

[29] J.M.R. Marín and H.K. Rasmussen, Lagrangian Finite?Element Method for the Simulation of K-BKZ Fluids with Third Order Accuracy, Journal of Non-Newtonian Fluid Mechanics 156, 177-188 (2009).

[30] H.K. Rasmussen, Lagrangian viscoelastic flow computations using a generalized molecular stress function model, Journal of Non-Newtonian Fluid Mechanics 106, 107-120 (2002).

[31] R. Keunings, Finite Element Methods for Integral Viscoelastic Fluids, in Rheology Reviews 2003, D.M. Binding and K. Walters (Eds.), British Society of Rheology, 167-195 (2003).

[32] E.A.J.F. Peters, M.A. Hulsen, and B.H.A.A. van den Brule, Instationary Eulerian viscoelastic flow simulations using time separable Rivlin-Sawyers constitutive equations, Journal of NonNewtonian Fluid Mechanics 89, 209-228 (2000).

[33] M.A. Hulsen, E.A.J.F. Peters, and B.H.A.A. van den Brule, A new approach to the deformation fields method for solving complex flows using integral constitutive equations, Journal of NonNewtonian Fluid Mechanics 98, 201-211 (2001). 
[34] P. Wapperom and R. Keunings, Simulation of linear polymer melts in transient complex flow, Journal of Non-Newtonian Fluid Mechanics 95, 67-83 (2000).

[35] P. Wapperom and R. Keunings, Numerical simulation of branched polymer melts in transient complex flow using pom-pom models, Journal of Non-Newtonian Fluid Mechanics 97, 267-281 (2001).

[36] R.D. Egholm, S.F. Christensen, and P. Szabo, Stress-strain behaviour in uniaxial compression of polymer gel beads, Journal of Applied Polymer Science 102, 3037-3047 (2006).

[37] M.F. Tome, M.S.B. de Araujo, M.A. Alves, and F.T. Pinho, Numerical simulation of viscoelastic flows using integral constitutive equations: A finite difference approach, Journal of Computational Physics 227, 4207-4243 (2008).

[38] M.F. Tome, J. Bertoco, C.M. Oishi, M.S.B. Araujo, D. Cruz, F.T. Pinho, and M. Vynnycky, A finite difference technique for solving a time strain separable K-BKZ constitutive equation for two-dimensional moving free surface flows, Journal of Computational Physics 311, 114-141 (2016).

[39] P. Olley and M.H. Wagner, A modification of the convective constraint release mechanism in the molecular stress function model giving enhanced vortex growth, Journal of Non-Newtonian Fluid Mechanics 135 68-81 (2006).

[40] R.B. Bird, R.C. Armstrong, and O. Hassager, Dynamics of Polymeric Liquids, Vol I, Wiley, New York, 2nd edition, 1987.

[41] H. Lamb, Hydrodynamics, Cambridge University Press, Cambridge, 6th edition, 1932.

[42] O. Zienkiewicz and K. Morgan, Finite Elements and Approximation, Wiley/Interscience, New York, 1983.

[43] P. Keast, Moderate degree tetrahedral quadrature formulas, Computer Methods in Applied Mechanics and Engineering 55, 339-348 (1986).

[44] O. Schenk and K. Gärtner, Solving unsymmetric sparse systems of linear equations with PARDISO, Journal of Future Generation Computer Systems 20, 475-487 (2004).

[45] O. Schenk and K. Gärtner, On fast factorization pivoting methods for symmetric indefinite systems. Electronic Transaction on Numerical Analysis 23, 158-179 (2006).

[46] X.L. Luo and R.I. Tanner, Finite element simulation of long and short circular die extrusion experiments using integral models, International Journal for Numerical Methods in Engineering 25, 9-22 1988.

[47] G. Barakos and E. Mitsoulis, A convergence study for the numerical simulation of the IUPACLDPE extrusion experiments, Journal of Non-Newtonian Fluid Mechanics 58, 315-329 (1995).

[48] A. Bach, H.K. Rasmussen, P-Y. Longin, and O. Hassager, Growth of non-axisymmetric disturbances of the free surface in the filament stretching rheometer: experiments and simulation, Journal of Non-Newtonian Fluid Mechanics 108, 163-186 (2002).

[49] H.K. Rasmussen and K. Yu, Spontaneous breakup of extended monodisperse polymer melts, Physical Review Letters 107, 126001 (2011). 
[50] H.K. Rasmussen, Catastrophic failure of polymer melts during extension, Journal of NonNewtonian Fluid Mechanics 198, 136-140 (2013).

[51] P.K. Currie, Constitutive equations for polymer melts predicted by the Doi-Edwards and CurtissBird kinetic theory models, Journal of Non-Newtonian Fluid Mechanics 11, 53-68 (1982).

[52] M.H. Wagner and V.H. Rolón-Garrido, The interchain pressure effect in shear rheology, Rheologica Acta 49, 459-471 (2010).

[53] M. Baumgaertel, A. Schausberger, and H.H. Winter, The relaxation of polymers with linear flexible chains of uniform length, Rheologica Acta 29, 400-408 (1990).

[54] C. Geuzaine and J.-F. Remacle, Gmsh: A 3-D finite element mesh generator with built-in preand post-processing facilities, International Journal of Numerical Methods in Engineering 79, 1309-1331 (2009). 